\title{
Estudo da adsorção de poluentes orgânicos em materiais poliméricos
}

Study of organic pollutants adsorption in polymeric materials

\author{
S. F. Amarante ${ }^{1 *}$; C. A. B. Garcia' ; C. D.A. E. S. Barbosa ${ }^{3}$ \\ ${ }^{1}$ Departamento de Engenharia Química, Universidade Federal de Sergipe, 49.100-000, São Cristóvão-Sergipe, Brasil \\ ${ }^{2}$ Departamento de Química, Universidade Federal de Sergipe, 49.100-000, São Cristóvão-Sergipe-Brasil \\ ${ }^{3}$ Departamento de Química, Universidade Federal de Pernambuco, 50.670-901, Recife-Pernambuco-Brasil \\ *simonise_fa@hotmail.com \\ (Recebido em 02 de março de 2016; aceito em 16 de abril de 2016)
}

\begin{abstract}
A poluição de cursos d'água por efluentes contendo corantes gera grande preocupação. Até o presente momento não há um método geral para a descontaminação de efluentes aquosos da indústria têxtil. $\mathrm{O}$ processo de adsorção vem se destacando no setor industrial, devido, principalmente, ao baixo custo e eficiência nas taxas de remoção. Neste estudo, a adsorção de corantes industriais por esferas de quitosana mostrou que o aumento da concentração do corante Turquesa G 133\% Remazol aumenta a capacidade adsortiva das esferas. Testes cinéticos com amostra de efluente real demonstraram que a capacidade de adsorção do material foi menor do que a observada com o uso do corante sintético, entretanto, após 24 horas verificou-se uma queda significativa da absorbância da amostra, mostrando que o material sintetizado é promissor.
\end{abstract}

Palavras-chave: adsorção, corantes, esferas de quitosana

Pollution of waterways by effluents containing dyes generates great concern. To date there is no general method for the decontamination of wastewater from the textile industry. The adsorption process has been highlighted in the industrial sector, mainly due to the low cost and efficiency in removal rates. In this study, the adsorption of dyes for industrial chitosan beads showed that the concentration of the colorant Remazol Turquoise G 133\% increases the adsorptive capacity of the beads. Kinetic tests with real effluent sample demonstrated that the adsorption capacity of the material was smaller than that observed with the use of synthetic dye, however, after 24 hours there was a significant drop of sample absorbance, showing that the synthesized material is promising.

Keywords: Adsorption, dyes, beads of chitosan.

\section{INTRODUÇÃO}

Tendo como foco a industrialização a poluição gerada com ela se torna também preocupante, o que não seria diferente para indústrias têxteis, que têm por grande dificuldade o controle e a remoção dos corantes de efluentes residuais [1]. Mesmo em pequenas concentrações, corantes nos cursos d'água são inaceitáveis por razões estéticas, sua reatividade e permanência no meio ambiente, afetando a vida aquática e a cadeia alimentar, alguns causando até dermatite alérgica e irritação da pele [2].

Até o presente momento não há um método geral para a descontaminação de efluentes aquosos da indústria têxtil. A maioria das indústrias realiza processos de oxidação biológica (lodo ativado) que não é efetivo na remoção da cor de muitos tipos de efluentes [3,4]. Existem muitos métodos físicos ou químicos para remoção de corantes que incluem coagulação, floculação, oxidação, fotodegradação, membrana filtrante, incluindo degradação biológica aeróbia e anaeróbia, mas todos esses métodos possuem limitações e nenhum deles é completamente satisfatório na remoção da cor de efluentes têxteis [5-8]. O processo de adsorção vem se destacando no setor industrial, devido, principalmente, ao baixo custo e eficiência nas taxas de remoção [9].

A remoção de corantes de efluentes têxteis por adsorção tem sido bastante pesquisada e relatada em vários trabalhos, tendo como principal vantagem à possibilidade de recuperação do corante na forma concentrada e a reutilização do adsorvente no processo [10,11].

Uma variedade de adsorventes tem sido empregada, destacando-se carvão ativo [12], turfa, sílica [13], alumina, celulose e, mais recentemente, quitina e quitosana [14]. 
A quitosana é um poliaminossacarídeo, em forma configuracuinal de um copolímero linear, constituído por unidades de poli $(1 \rightarrow 4) 2$ - amino- 2 - deoxi- $\beta$ - D- glucopiranose (principalmente) e poli $(1 \rightarrow 4) 2$ - acetanamida- 2 - deoxi- $\beta$ - D- glucopiranose $[15,16]$. É preparada, usualmente, por purificação de $\mathrm{N}$ - desacetilação da quitina em meio básico [17]. Tanto a quitina quanto a quitosana são materiais biologicamente inertes e não apresentam perigo ao ser humano, nem ao meio ambiente, o que faz delas produtos vantajosos para vários fins, dentre eles o industrial. Como vantagens estão a não toxicidade, biodegradação, polifuncionalidade, alta reatividade química, capacidades de adsorção $[18,19]$.

Para maior estabilidade e melhoramento das propriedades mecânicas das esferas de quitosana, utiliza-se o processo de reticulação, que além do fortalecimento do material, propicia também a não dissolução em meio ácido quando se utiliza reticulantes à base de grupos fosfatos [20,21]. Esse método consiste na introdução de moléculas de baixa massa molar, chamados de agentes de reticulação. Os grupos funcionais $(-\mathrm{OH},-\mathrm{COOH},-\mathrm{NH} 2)$ na estrutura dos polissacarídeos formam ligações cruzadas com moléculas bifuncionais ou íons, os quais permitem a formação de pontes entre duas cadeias da macromolécula. Dependendo do agente de reticulação essas interações podem ser covalentes ou iônicas [22].

O presente trabalho é um estudo do processo de adsorção de corantes utilizando como material adsorvente esfera de quitosana precipitada em solução de hidróxido de sódio, reticuladas em solução de tetrapirofosfato de sódio, em sistema sintético (corante Turquesa G 133\% Remazol) e real.

\section{MATERIAL E MÉTODOS}

\subsection{Reagentes e Soluções}

A quitosana em pó utilizada neste trabalho foi da marca Polymar, com grau de desacetilação $85,0 \%$. O corante Turqueza G 133\% Remazol e a amostra real do efluente foram cedidos por uma indústria têxtil localizada no estado de Sergipe. Os reagentes utilizados foram ácido acético (Merck), hidróxido de sódio (Vetec Química Fina) e tetrapirofosfato de sódio (Merck), todos padrão analítico.

\subsection{Espectroscopia de infravermelho}

Espectroscopia na região do infravermelho é uma das técnicas mais comuns e utilizadas em diversas áreas. Baseia-se fundamentalmente na medida da absorção de energia em freqüências de infravermelho por uma amostra posicionada no caminho do feixe de radiação infravermelha. As radiações infravermelhas apresentam comprimentos de onda típicos que variam de 0,78 a

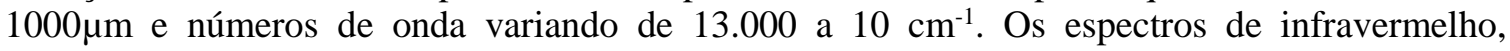
geralmente, são gráficos construídos na forma de número de onda ou comprimento de onda (eixo $\mathrm{X})$ versus absorbância ou transmitância (eixo Y) [23].

O objetivo desta técnica é a determinação dos grupos funcionais presentes na estrutura dos catalisadores. Cada grupo absorve em frequência característica de radiação na região do infravermelho (IV). Assim, um gráfico de intensidade de radiação versus frequência (espectrograma de IV), permite caracterizar os grupos funcionais de um padrão ou de um material desconhecido.

As análises foram realizadas utilizando pastilha de $\mathrm{KBr}$, em um equipamento da Perkin Elmer Spectrum BX, com resolução de $4 \mathrm{~cm}^{-1}$, na faixa de $4000-400 \mathrm{~cm}^{-1}$ com 16 acumulações.

\subsection{Preparação das esferas de quitosana}

$5 \mathrm{~g}$ de quitosana foram dissolvidos em $100 \mathrm{~mL}$ de uma solução de ácido acético $(3 \% \mathrm{~m} / \mathrm{v})$. O gel obtido foi precipitado sobre solução de $\mathrm{NaOH} 6 \%(\mathrm{~m} / \mathrm{v})$, com o auxílio de uma bureta. As esferas de quitosana formadas permaneceram em contato com o precipitante sob agitação 
mecânica, por $1 \mathrm{~h}$. Em seguida, foram lavadas com água ultrapura até atingir pH próximo da neutralidade.

\subsection{Reticulação das esferas de quitosana}

As esferas foram postas em contato com a solução reticulante de tetrapirofosfato de sódio $4 \%$ $(\mathrm{m} / \mathrm{v})$, durante 24 horas. Posteriormente foram lavadas até $\mathrm{pH}$ neutro e secas em estufa à $60^{\circ} \mathrm{C} . \mathrm{O}$ material adsorvente obtido foi denominado de QNP.

\subsection{Cinética de Adsorção}

$1 \mathrm{~g}$ de QNP foi posto em contato com $100 \mathrm{~mL}$ de uma soluções de corante Turquesa $\mathrm{G} 133 \%$ Remazol com diferentes concentrações $\left(5,10\right.$ e $\left.20 \mathrm{mg} . \mathrm{L}^{-1}\right)$. O sistema foi mantido a $25 \pm 2{ }^{\circ} \mathrm{C}$,

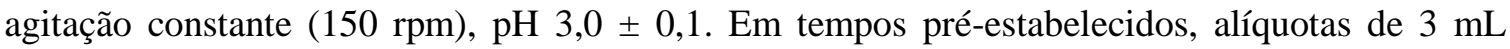
foram retiradas do contato com as esferas, com auxílio de uma pipeta automática, e analisadas em um espectofotômetro (Biochrom, Libra S12) a $661 \mathrm{~nm}$. A capacidade de adsorção do adsorvente, $Q$ (mg do metal/g do adsorvente), foi determinada com base na diferença da concentração do corante, usando-se a Equação 1.

$$
Q=\frac{\left(C_{0}-C_{f}\right) \cdot V}{m}
$$

onde $\mathrm{C}_{0}$ é concentração inicial $\left(\mathrm{mg} . \mathrm{L}^{-1}\right) ; \mathrm{C}_{\mathrm{f}}$ é concentração final $\left(\mathrm{mg} \cdot \mathrm{L}^{-1}\right)$; $\mathrm{V}$ é volume da solução (L); m é massa do adsorvente (g).

\subsection{Teste com amostra de efluente real}

Para realização deste teste, $100 \mathrm{~mL}$ do efluente foi colocado em contato com $1 \mathrm{~g}$ de QNP. Em intervalos de tempo pré-estabelecidos, alíquotas de $3 \mathrm{~mL}$ foram retiradas do sistema e varreduras no espectrofotômetro de 300 a $700 \mathrm{~nm}$ foram realizadas entre os intervalos de tempo pré-estabelecidos, até que o equilíbrio fosse estabelecido, em aproximadamente 24 horas.

\section{RESULTADOS E DISCUSSÃO}

A composição química do pó e das esferas de quitosana foi investigada por espectroscopia na região do infravermelho e seu espectro é mostrado na Figura 1.

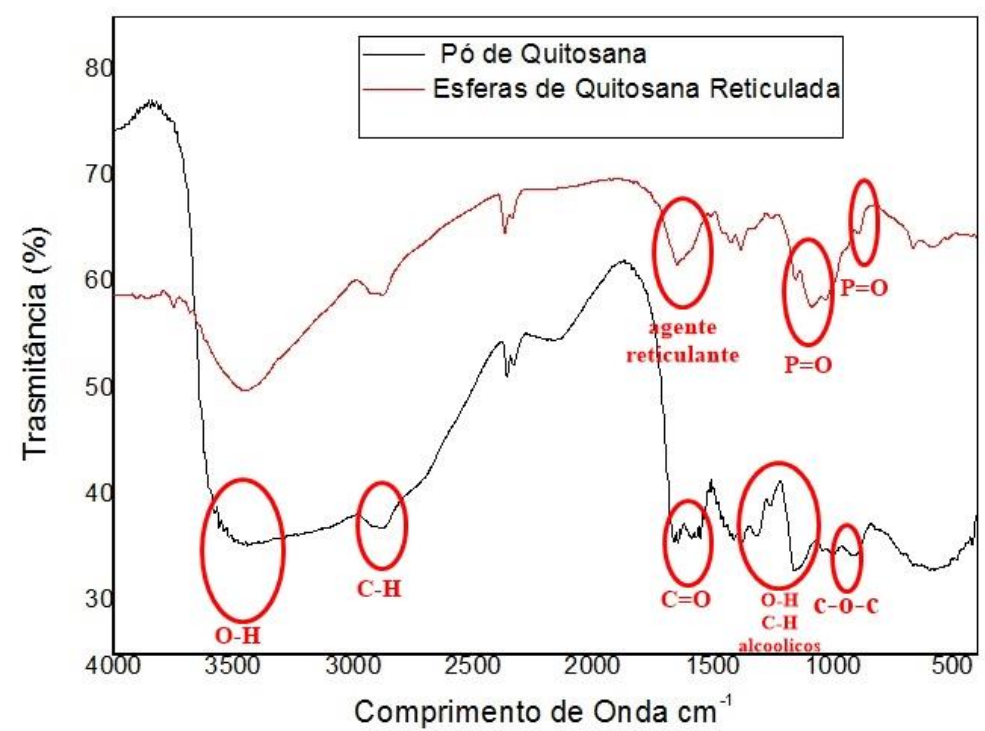

Figura 1: Espectros de transmissão na região do infravermelho do pó de quitosana e das esferas de quitosana reticulada. 
A quitosana apresenta uma banda larga, entre 3628 e $3455 \mathrm{~cm}^{-1}$ ocasionada pelo estiramento axial de $\mathrm{O}-\mathrm{H}$, sobreposto a vibração de deformação axial de $\mathrm{N}-\mathrm{H}$. As vibrações observadas em torno $2888 \mathrm{~cm}^{-1}$ correspondem a estiramentos de C-H. A banda de absorção encontrada em $1652 \mathrm{~cm}^{-1}$ corresponde as vibrações de estiramento $\mathrm{C}=\mathrm{O}$ de amida secundária, sendo uma contribuição da quitina, em $1552 \mathrm{~cm}^{-1}$ encontra-se vibrações geradas pela deformação angular de $\mathrm{NH}_{2}$ [24]. Deformações angulares de $\mathrm{O}-\mathrm{H}$ acoplado a deformações angulares de C-H provenientes álcoois primários são encontradas em 1427 e $1388 \mathrm{~cm}^{-1}$ respectivamente [25]. Na faixa compreendida entre 1152 e $890 \mathrm{~cm}^{-1}$ encontram-se bandas vibracionais de estruturas polissacarídicas, tais como deformação de C-O acoplado com vibrações axiais de C-C em 1152 e $1070 \mathrm{~cm}^{-1}$ e deformação axial simétrica de C-O-C em $1030 \mathrm{~cm}^{-1}$.

No espectro das esferas, supõe-se que a interação do agente reticulante ocorre através dos grupos amino protonados $\left(\mathrm{NH}_{3}{ }^{+}\right)$da quitosana, portanto, uma interação iônica pode ser evidenciada na região próxima a $1552 \mathrm{~cm}^{-1}$. As bandas em 1228 e $889 \mathrm{~cm}^{-1}$ estão relacionadas às vibrações de estiramento do grupo $\mathrm{P}=\mathrm{O}$. Pelo exposto podem-se perceber mudanças no material precusor (quitosana pura) e naquele sintetizado (esferas de quitosana), evidenciando a existência de um material modificado [25].

A cinética de adsorção do corante Turquesa G 133\% Remazol pelas esferas de QNP, Figura 2, mostra que o tempo de equilíbrio para o material QNP foi de aproximadamente 1000 min para a concentração de $20 \mathrm{mg} . \mathrm{L}^{-1}$, e de $480 \mathrm{~min}$ para as concentrações de 5 e $10 \mathrm{mg} . \mathrm{L}^{-1}$.

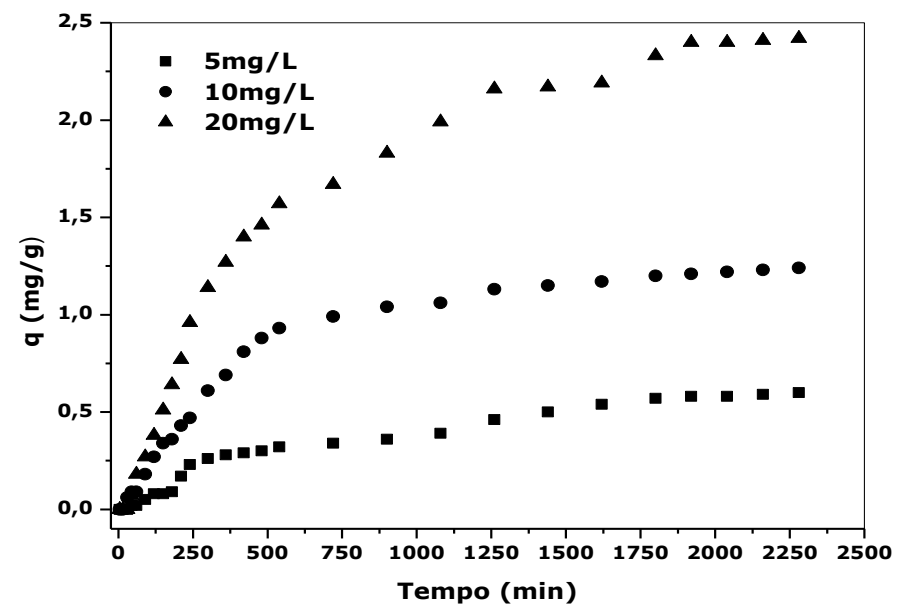

Figura 2: Isoterma de equilíbrio de adsorção do corante Turquesa G 133\% Remazol em esferas de quitosana/NaOH reticuladas em pirofosfato de sódio $(p h \approx 3,100 \mathrm{~mL}, 1 \mathrm{~g})$.

Os resultados obtidos, na análise cinética do corante Turquesa, mostram que a adsorção do corante pela QNP ocorre de forma progressiva. As curvas cinéticas também evidenciaram que a capacidade de adsorção aumenta com o tempo até atingir um valor constante, onde nenhuma adsorção adicional acontece [26]. Foi observado que os gráficos cinéticos de adsorção do corante pela QNP mostraram de forma geral duas fases de adsorção. A primeira é rápida e acontece até cerca de $1000 \mathrm{~min}$ para a concentração de $20 \mathrm{mg} . \mathrm{L}^{-1}$, e $500 \mathrm{~min}$ para as concentrações de 5 e 10 mg. $\mathrm{L}^{-1}$. Uma segunda etapa, mais lenta, ocorre ao término da etapa comentada anteriormente, nesta segunda o equilíbrio é alcançado. A primeira etapa está, normalmente, relacionada a uma adsorção na superfície externa do adsorvente, que acontece de maneira geral, instantaneamente ou em tempos relativamente curtos. A segunda ocorre de forma gradual antes do corante alcançar o equilíbrio. A alta capacidade inicial de adsorção do corante pela QNP pode estar relacionada também à existência de uma maior quantidade de sítios ativos expostos na superfície do adsorvente no início do processo [27].

No teste cinético com amostra de efluente real, Figura 3, a capacidade adsortiva das esferas de quitosana foi menor do que com o corante sintético, isso pode ser explicado pelo fato de o efluente real ter apresentado um $\mathrm{pH}$ em torno de 10, podendo ter ocorrido uma menor 
contribuição na interação do material adsorvente com a amostra. Entretanto, em uma avaliação geral da varredura do efluente, foi observado que em $24 \mathrm{~h}$ de contato com as QNP ocorreu uma redução significativa na absorbância do efluente, comprovando assim que o material é promissor.

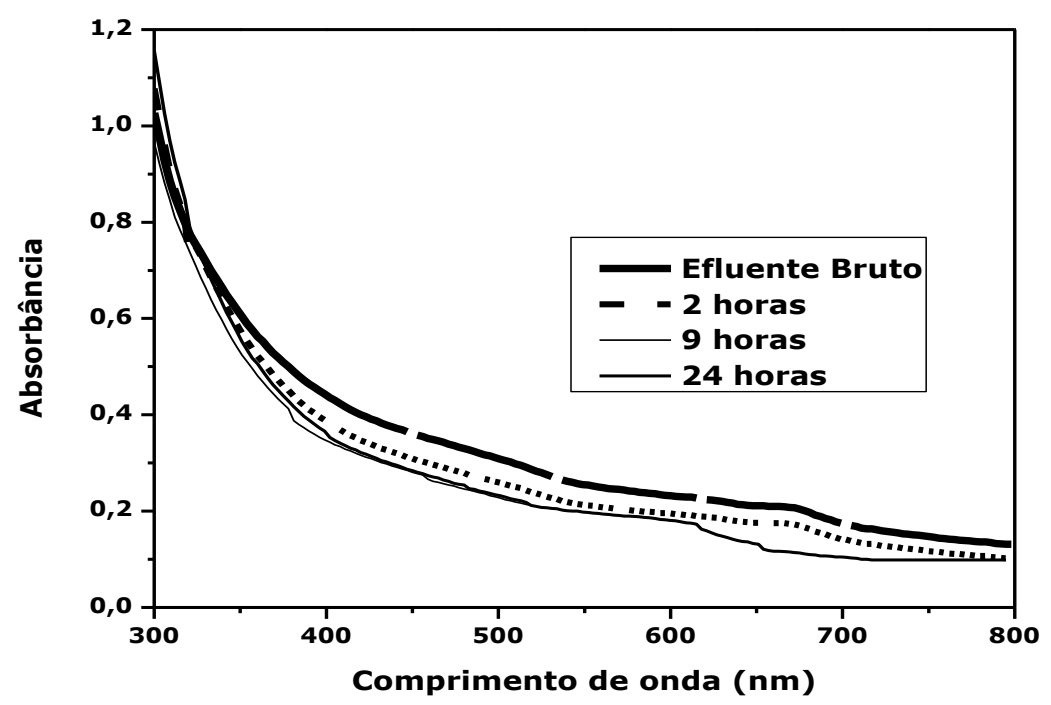

Figura 3: Varredura amostra real em contato com QNP.

\section{CONCLUSÃO}

Neste trabalho, realizaram-se testes de adsorção do corante sintético Turquesa G $133 \%$ Remazol e com efluente indutrial têxtil utilizando como material adsorvente esferas de quitosana precipitadas sobre Hidróxido de Sódio e reticuladas com tetrapirofosfato de sódio. Através da caracterização do adsorvente por espectroscopia de infravermelho (FITR) foi possível observar e sugerir interações da quitosana com o reticulante. As curvas cinéticas evidenciaram que a capacidade de adsorção aumenta com o tempo de contato e esses valores foram maiores com o aumento da concentração inicial. No teste com amostra de efluente real a capacidade adsortiva do material foi menor do que com o corante sintético, porém, ficou evidente que a QNP pode ser considerada um material promissor para uso em escala industrial, como, por exemplo, para o tratamento de efluentes em estações de tratamento em indústrias têxteis.

\section{AGRADECIMENTOS}

Ao Laboratório de Química Analítica Ambiental da Universidade Federal de Sergipe.

\section{REFERÊNCIAS BIBLIOGRÁFICAS}

1. Pinheiro HM, Touraud E, Thomas O. Aromatic amines from azo dye reduction: status review with emphasis on direct UV spectrophotometric detection in textile industry wastewaters. Dyes Pigm. 2004 Mai, 61(2), 121-139, doi:10.1016/j.dyepig.2003.10.009.

2. Mechichi T, Mhiri N, Sayadi S. Remazol Brilliant Blue R decolourization by the laccase from Trametes trogii. Chemosphere. 2006 Ago, 64(6), 998-1005, doi:10.1016/j.chemosphere.2005.12.061.

3. Eichlerová I, Homolka L, Nerud F. Decolorization of high concentrations of synthetic dyes by the white rot fungus Bjerkandera adusta strain CCBAS 232. Dyes Pigm. 2007 Mai, 75(1), 38-44, doi:10.1016/j.dyepig.2006.05.008. 
4. García-Montaño J, Domènech X, García-Hortal JA, Torrades F, Peral J. The testing of several biological and chemical coupled treatments for Cibacron Red FN-R azo dye removal. J. Hazard. Mater. 2008 Jun, 154(1-3), 484-490, doi:10.1016/j.jhazmat.2007.10.050.

5. Oztürk A, Abdullah MI. Toxicological effect of indole and its azo dye derivatives on some microorganisms under aerobic conditions. Sci. Total Environ. 2006 Abr, 358(1-3), 137-142, doi:10.1016/j.scitotenv.2005.08.004.

6. Neamtu M, Yediler A, Siminiceanu I, Kettrup A. Decolorization of Disperse Red 354 Azo Dye in Water by Several Oxidation Processes - A Comparative Study. Dyes Pigm. 2004 Jun, 60, 61-68, doi:10.1016/S0143-7208(03)00129-3.

7. Chakraborty S, Purkait MK, DasGupta S, De S, Basu JK. Nanofiltration of textile plant effluent for color removal and reduction in COD. Sep. Purif. Technol. 2003 May, 31(2), 141-151, doi:10.1016/S1383-5866(02)00177-6.

8. Feifang Z, Ayfer Y, Xinmiao L, Antonius K. Effects of dye additives on the ozonation process and oxidation byproducts: a comparative study using hydrolyzed CI Reactive red 120. Dyes Pigm. 2004 Jan, 60(1), 1-7, doi:10.1016/S0143-7208(03)00111-6.

9. Twardokus RG. Reuso de Água no Processo de Tingimento da Indústria Têxtil [dissertação], Florianópolis (SC): Universidade Federal de Santa Catarina, 2004, 136 p.

10. Darinka BV, Alenka MLM. Reactive dye decolorization using combined ultrasound $/ \mathrm{H}_{2} \mathrm{O}_{2}$. Dyes Pigm. 2003 Nov, 59(2), 173-179, doi:10.1016/S0143-7208(03)00101-3.

11. Quadros SS. Tratamento e reutilização de efluentes têxteis gerados nos tingimentos de tecidos de algodão [dissertação], Blumenau (SC): Universidade Regional de Blumenau, 2005, 105 p.

12. Mussatos SI, Roberto IC. Hydrolysate detoxification with activated charcoal for xylitol production by Candida guilliermondii. Biotechnol. Lett. 2001 Out, 23(20), 1681-1684, 10.1023/A:1012492028646.

13. Jal PK, Patel S, Mishra BK. Chemical modification of silica surface by immobilization of functional groups for extractive concentration of metal ions. Talanta. 2004 Abr, 62(5), 1005-1028, doi:10.1016/j.talanta.2003.10.028.

14. Nawar SS, Doma HS. Removal of dyes from effluents using low-cost agricultural by-products. Sci. Total Environ. 1989 Abr, 79(3), 271-279, doi:10.1016/0048-9697(89)90342-2.

15. Alves NM, Mano JF. Chitosan derivatives obtained by chemical modifications for biomedical and environmental applications. Int. J. Biol. Macromol. 2008 Dez, 43(5), 401-414, doi:10.1016/j.ijbiomac.2008.09.007.

16. Monteiro Júnior OAC, Airoldi C. The influence of chitosans with defined degrees of acetylation on the thermodynamic data for copper coordination. J. Colloid Interface Sci. 2005 Fev, 282(1), 3237, doi:10.1016/j.jcis.2004.08.165.

17. Cestari AR, Vieira EF, Dos Santos AG, Mota JA, De Almeida VP. Adsorption of anionic dyes on chitosan beads. 1. The influence of the chemical structures of dyes and temperature on the adsorption kinetics. J. Colloid Interface Sci. 2004 Dez, 280(2), 380-386, doi:10.1016/j.jcis.2004.08.007.

18. Crini G, Badot PM. Application of chitosan, a natural aminopolysaccharide, for dye removal from aqueous solutions by adsorption processes using batch studies: A review of recent literature. Prog. Polym. Sci. 2008 Abr, 33(4), 399-447, doi:10.1016/j.progpolymsci.2007.11.001.

19. Barros FCF, Cavalcante RM, Carvalho TV, Dias FS, Queiroz DC, Vasconcelos LCG, Nascimento RF. Produção e caracterização de esfera de quitosana modificada quimicamente. R. Iberoamer. Polím. 2006, 7(4), 232-246.

20. Lee ST, Mi FL, Shen YJ, Shyu SS. Equilibrium and kinetic studies of copper(II) ion uptake by chitosan-tripolyphosphate chelating resin. Polymer. 2001 Mar, 42(5), 1879-1892, doi:10.1016/S0032-3861(00)00402-X.

21. Yi Y, Wang Y, Liu H. Preparation of new crosslinked chitosan with crown ether and their adsorption for silver ion for antibacterial activities. Carbohydr. Polym. 2003 Set, 53(4), 425-430, doi:10.1016/S0144-8617(03)00104-8.

22. Hirata S, Kajiya T, Takano N, Aihara M, Honda K, Shikino O, Nakayama E. Determination of trace metals in seawater by on-line column preconcentration inductively coupled plasma mass spectrometry using metal alkoxide glass immobilized 8-quinolinol. Anal. Chim. Acta. 2003 Dez, 157(1-2), 157-165, doi:10.1016/S0003-2670(03)00949-8.

23. Settle F. Handbook of instrumental techniques for analytical chemistry. New York: Prentice Hall, 1997. 995p.

24. Vaghetti JCP, Zat M, Bentes KRS, Ferreira LS, Benvenutti EV, Lima EC. 4Phenylenediaminepropylsilica xerogel as a sorbent for copper determination in waters by slurrysampling ETAAS. J. Anal At. Spectrom. 2003 Fev, 18, 376-380, doi: 10.1039/b209133j. 
25. Laus R, Laranjeira MCM, Martins AO, Fávere VT, Pedrosa RC, Benassi JC, Geremias R. Microesferas de quitosana reticuladas com tripolifosfato utilizadas para remoção da acidez, ferro(iii) e manganês(ii) de águas contaminadas pela mineração de carvão. Quim. Nova. 2006 Dez, 29(1), 34-39.

26. Amuda OS, Giwa AA, Bello IA. Removal of heavy metal from industrial wastewater using modified activated coconut shell carbon. Bioch. Eng. J. 2007 Set, 36(2), 174-181, doi:10.1016/j.bej.2007.02.013 .

27. Baldissarelli VZ. Estudo da adsorção do corante reativo preto 5 sobre carvão ativado: caracterização do adsorvente e determinação de parâmetros cinéticos e termodinâmicos [dissertação], Blumenau (SC): Universidade Regional de Blumenau, 2006, 117 p. 